\title{
The Clinicopathological features and survival outcomes of patients with different metastatic sites in stage IV breast cancer
}

\author{
Ru Wang ${ }^{1,2}$, Yayun Zhu ${ }^{2,3}$, Xiaoxu Liu' ${ }^{1}$, Xiaoqin Liao ${ }^{1}$, Jianjun $\mathrm{He}^{1}$ and Ligang $\mathrm{Niu}^{1 *}$ (1)
}

\begin{abstract}
Background: The features and survival of stage IV breast cancer patients with different metastatic sites are poorly understood. This study aims to examine the clinicopathological features and survival of stage IV breast cancer patients according to different metastatic sites.

Methods: Using the Surveillance, Epidemiology, and End Results database, we restricted our study population to stage IV breast cancer patients diagnosed between 2010 to 2015. The clinicopathological features were examined by chi-square tests. Breast cancer-specific survival (BCSS) and overall survival (OS) were compared among patients with different metastatic sites by the Kaplan-Meier method with log-rank test. Univariable and multivariable analyses were also performed using the Cox proportional hazard model to identify statistically significant prognostic factors.

Results: A total of 18,322 patients were identified for survival analysis. Bone-only metastasis accounted for 39.80\% of patients, followed by multiple metastasis (33.07\%), lung metastasis (10.94\%), liver metastasis (7.34\%), other metastasis (7.34\%), and brain metastasis (1.51\%). The Kaplan-Meier plots showed that patients with bone metastasis had the best survival, while patients with brain metastasis had the worst survival in both BCSS and OS ( $p<0.001$, for both). Multivariable analyses showed that age, race, marital status, grade, tumor subtype, tumor size, surgery of primary cancer, and a history of radiotherapy or chemotherapy were independent prognostic factors.

Conclusion: Stage IV breast cancer patients have different clinicopathological characteristics and survival outcomes according to different metastatic sites. Patients with bone metastasis have the best prognosis, and brain metastasis is the most aggressive subgroup.
\end{abstract}

Keywords: Breast cancer, Metastatic sites, SEER, Survival outcomes

\section{Background}

Breast cancer $(\mathrm{BC})$, the most common malignancy in women, is also the key contributor to cancer-related deaths worldwide in female. 2.08 million women were diagnosed with breast cancer in 2018, occupying $24.2 \%$ of all new cancer cases in that year, according to the 2018 global cancer statistics. In addition, 626,679 women are expected to die from this disease, making up $15 \%$ of all cancer deaths among females [1]. Despite the high morbidity of the disease, patients with breast cancer have better prognosis compared to other aggressive

\footnotetext{
* Correspondence: chinanlg@163.com

'Department of Breast Surgery, The First Affiliated Hospital of Xi'an Jiaotong

University, 277 West Yanta Road, Xi'an 710061, Shaanxi, China

Full list of author information is available at the end of the article
}

cancers. According to the American Cancer Society's biennial update on female breast cancer statistics, the 5year survival rate is $91 \%$ and 10 -year survival rate is $84 \%$ [2]. However, the survival decreases greatly if the patients develop distant metastases. The overall 5-year relative survival rate is $99 \%$ for localized diseases and $86 \%$ for regional diseases, which drops to $27 \%$ for distant-stage diseases [3].

It is estimated that $20-30 \%$ of early stage breast cancers will go on to develop metastatic disease, and 6-10\% of all women suffering from breast cancer in the United States were found to have stage IV disease at diagnosis (called de novo metastatic breast cancer) [4]. These patients have poor prognoses with a median survival time of 2-3 years [5], despite technology development and

(c) The Author(s). 2019 Open Access This article is distributed under the terms of the Creative Commons Attribution 4.0 International License (http://creativecommons.org/licenses/by/4.0/), which permits unrestricted use, distribution, and reproduction in any medium, provided you give appropriate credit to the original author(s) and the source, provide a link to the Creative Commons license, and indicate if changes were made. The Creative Commons Public Domain Dedication waiver (http://creativecommons.org/publicdomain/zero/1.0/) applies to the data made available in this article, unless otherwise stated. 
medical progresses that have been made nowadays. Metastatic breast cancer is a heterogeneous disease with distinct prognoses [6], which are affected by many clinicopathological features of patients, such as age, race, marital status, performance status as well as tumor size, lymph nodes status, metastatic sites, number of metastatic sites, pathological or genotype characteristics and previous medical treatments [7-9]. Therefore, an accurate estimation of survival may benefit patients significantly in decision-making [10]. The TNM stage is the widely accepted tool to predict the prognosis of patients, but it deals with a limited range of factors and ignores patient-specific conditions, pathological or genotype characteristics, and treatments [10]. Therefore, it is still difficult to make precise predictions about individual prognoses for metastatic breast cancer using this method. Among all the predictors of outcome mentioned above, the metastatic site may be the most important factor with the greatest significant impact on further treatment regimens.

Population-level estimates for prognosis among breast cancer patients with distant metastases are lacking, and the relationship between clinical-related factors and the exact patterns of distant metastasis is not well elaborated. And thus, the purposes of this study were to use the Surveillance, Epidemiology, and End Results (SEER) database to present clinicopathological features and survival estimates, and to investigate the prognostic impact of stage IV breast cancer patients based on their metastatic sites at the time of cancer diagnosis on a population-based level.

\section{Methods}

\section{Patients}

The recent version of the Surveillance, Epidemiology and End Results (SEER) 18 registries Custom Data (with additional treatment fields) was used as the data source for the present population-based investigation. SEER*Stat Software version 8.3.5 (https://seer.cancer.gov/seer stat/) (Information Management Service, Inc. Calverton, MD, USA) was used to generate the case listing. Maintained by the National Cancer Institute, the SEER program is the largest publicly available cancer dataset in the world, which consists of 18 population-based cancer registries and covers approximately $26 \%$ of the US population across several geographic regions [11]. Patients diagnosed with female breast cancer as the primary cancer from 2010 to 2015, with distant metastasis, were enrolled into the study. The tumors were classified based on their primary site of presentation and histology utilizing the International Classification of Disease for Oncology, Third Edition (ICD-O-3). Patients with unknown metastatic sites and survival were excluded. Of 19,913 women with a diagnosis of stage IV breast cancer between the year 2010 and 2015 included into the SEER Registry, 18,322 women were eligible for inclusion into the present study (Fig. 1, Flowchart). Eligible patients were grouped according to metastatic site. All procedures performed in studies involving human participants were in accordance with the 1964 Helsinki declaration and its later amendments, or comparable ethical standards. Because the SEER database is publicly accessible, this study does not require informed patient consent and was deemed exempt from review by the Ethics Committee of the First Affiliated Hospital of Xian Jiaotong University.

\section{Demographic and clinical variables}

The relationship between metastatic sites and clinical characteristics, including age at diagnosis, year of diagnosis, race, marital status, tumor grade, tumor size, nodal status, subtype, and treatment was analyzed. Initial metastatic sites were registered as single or multiple and were categorized as bone-only, lung-only, liver-only,

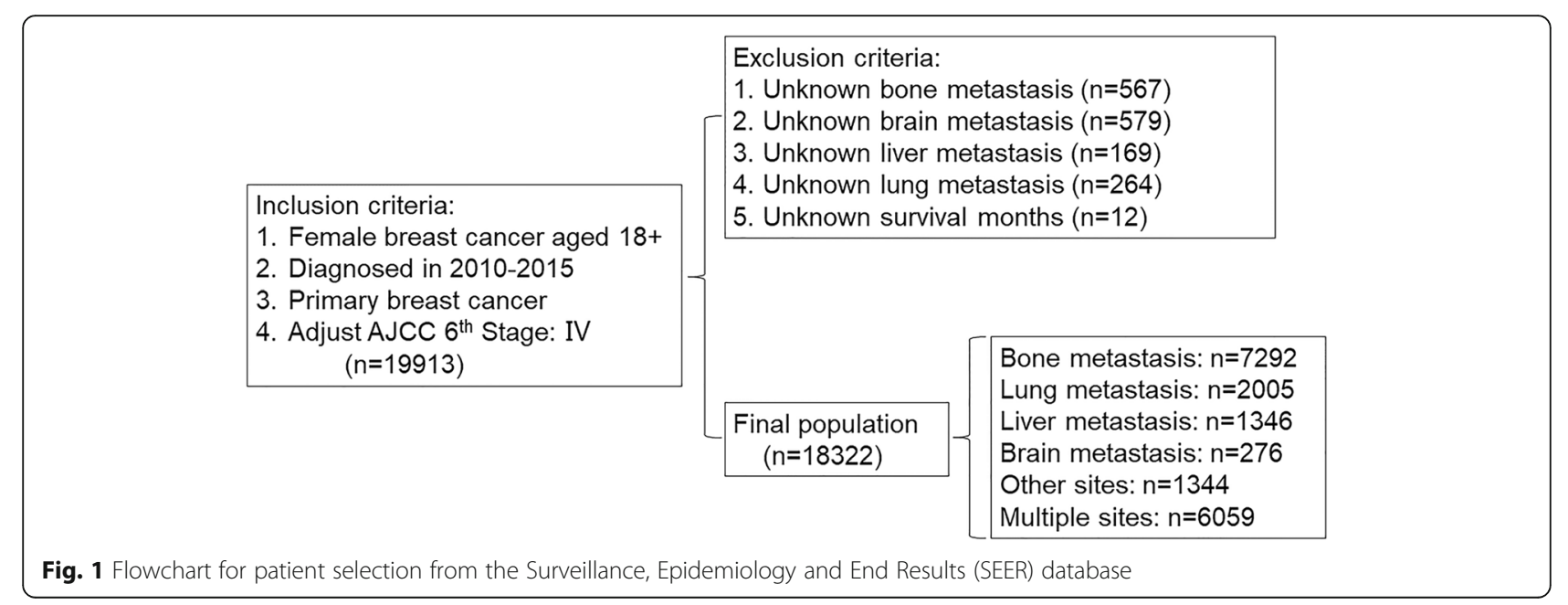


brain-only, other-only (metastatic sites other than the aforementioned sites such as skin and soft tissue) and multiple metastasis (more than one of the aforementioned distant metastatic sites). Overall survival (OS) was calculated from the date of diagnosis to the date of death due to any cause, the date of last follow-up, or December 31, 2015. Breast cancer specific survival (BCSS) was measured as the time from the date of diagnosis to the date of death attributed to breast cancer. Both overall survival and breast cancer specific survival were used as endpoints.

\section{Statistical analysis}

The baseline characteristics of patients and treatment were described using summary statistics, with continuous variables being shown as mean \pm standard deviation. Differences between qualitative variables and continuous variables were analyzed using $\chi^{2}$ statistics and analysis of variance, respectively. Survival was estimated using the Kaplan-Meier method and compared between the different metastatic groups using log-rank test. Hazard ratios (HRs) and 95\% confidence intervals (CIs) were calculated by univariable and multivariable Cox proportional hazard models to assess the relative contribution of metastatic sites and other factors to survival after diagnosis of metastatic breast cancer. The statistical analyses above were carried out using SPSS software version 22.0 (SPSS Inc.). Cumulative incidence function (CIF) was employed to show the probability of each competing event and the differences between the groups were estimated using Gray's test. Competing risk analysis were performed using $\mathrm{R}$ version 3.6.1 software (The $\mathrm{R}$ Foundation for Statistical Computing, Vienna, Austria; www.r-project.org) with the $\mathrm{R}$ packages cmprsk and survival. All the tests above were 2 -tailed, and a $p$ value of less than 0.05 was considered statistically significant.

\section{Results}

\section{Patient characteristics}

According to the above inclusion and exclusion criteria, a total of 18,322 patients were enrolled in this study. Table 1 shows baseline demographic and clinicopathological characteristics of the included patients according to metastatic sites. Median survival after diagnosis of metastatic disease was 26.0 months, with 8442 patients (46.1\%) being alive at the end of the follow-up period.

The largest subgroup was the bone metastasis, comprising $39.8 \%$ of all patients (7292), followed by multiple metastasis (33.07\%, 6059), lung metastasis (10.94\%, $2005)$, liver metastasis $(7.34 \%, 1346)$, other metastasis $(7.34 \%, 1344)$, and brain metastasis $(1.51 \%, 276)$. The median patient age at initial diagnosis was 62 years (range 20-100 years). The mean patient age for liver metastasis was the lowest (59.0 years), while lung metastasis was the highest (66.2 years) among all metastasis groups (range 61.2-64.3 years). Poorly or undifferentiated tumors were most common in liver metastasis (48.66\%), followed by lung metastasis $(47.16 \%)$ and brain metastasis (42.39\%). Patients with lung metastasis (50.69\%) and multiple metastases (48.07\%) tend to have larger tumors of T3-T4 at initial diagnosis. Furthermore, these two groups of patients also have a higher proportion of later $\mathrm{N}$ stage (N3), making up 29.48 and $33.94 \%$ respectively. The distribution of subtypes is significantly different between initial metastatic sites (Table 1). Bone was the predominant initial site of metastasis for the $\mathrm{HR}+/$ HER2- (64.4\%) group and the least common site in the HR-/HER2+ group (3.24\%). Of patients with brain, lung and liver metastasis, 26.9, 21.22, and $14.42 \%$, respectively, had the triple negative subtype. Detailed patient characteristics are presented in Table 1.

\section{Kaplan-Meier Survival analysis}

Of all the 18,322 patients finally recruited, 9880 patients had died by the end of the last follow-up, 7239 of whom died of breast cancer specifically. The median survival of all patients since diagnosis of distant metastases was 26.0 months. The median survival ranged from 8.0 months (95\% CI 5.65-10.35 months) for patients with brain metastasis to 36.0 months (95\% CI 34.74-37.27 months) for patients with bone metastasis.

The Kaplan-Meier plots were displayed in Fig. 2 to show the survival of all populations. Figure 2a shows the overall survival (OS) of stage IV patients who were enrolled into the study according to metastatic sites. Patients with bone metastasis had the best survival, with 3 -year OS rate of $50.5 \%$, followed by patients with other metastasis, liver metastasis, and lung metastasis, (with OS rate of $41.9,38.2$, and $37.5 \%$ respectively). Patients with brain metastasis and multiple metastasis had worse OS than other subgroups: the 3-year OS rate was 19.9, and $27.4 \%$, respectively $(p<0.001)$. Figure $2 \mathrm{~b}$ shows the breast cancer specific survival (BCSS) according to metastatic sites of stage IV patients. Similar to OS, brain metastasis had the worst survival, with a 3-year BCSS rate of $50.6 \%$. The 3-year BCSS rate with liver, lung, and multiple metastases were, 67.7, 66.1, and 62.6\% respectively. Bone and other metastasis had better BCSS, for which the 3-year BCSS rate was 76.6 and $65.3 \%$, respectively $(p<0.001)$.

Breast cancer subtype is an important prognostic factor since it affects survival significantly. The triple negative pattern tends to decrease survival dramatically compared to the other groups (Additional file 1: Figure S1). Survival estimates overall (Fig. 3) and breast cancer specific (Additional file 2: Figure S2) as stratified by subtype are graphically displayed in the Figure; HR+/HER2(A), HR+/HER2 + (B), HR-/HER2+ (C), and TN (D). In 
Table 1 Patient characteristics

\begin{tabular}{|c|c|c|c|c|c|c|c|c|}
\hline Characteristic & $n$ & $\begin{array}{l}\text { Bone } \\
\text { Metastasis (\%) }\end{array}$ & $\begin{array}{l}\text { Lung } \\
\text { Metastasis (\%) }\end{array}$ & $\begin{array}{l}\text { Liver } \\
\text { Metastasis (\%) }\end{array}$ & $\begin{array}{l}\text { Brain } \\
\text { Metastasis (\%) }\end{array}$ & $\begin{array}{l}\text { Other Metastasis } \\
\text { sites (\%) }\end{array}$ & $\begin{array}{l}\text { Multiple } \\
\text { Metastasis sites (\%) }\end{array}$ & $p$ \\
\hline$n$ & 18,322 & $7292(39.80)$ & 2005(10.94) & $1346(7.34)$ & $276(1.51)$ & $1344(7.34)$ & 6059(33.07) & \\
\hline Age (years, mean $\pm S D$ ) & $62.34 \pm 14.4$ & $62.47 \pm 14.2$ & $66.24 \pm 14.9$ & $59.01 \pm 15.1$ & $61.15 \pm 14.1$ & $64.34 \pm 14.5$ & $61.25 \pm 13.8$ & $<0.001$ \\
\hline \multicolumn{9}{|l|}{ Year of diagnosis } \\
\hline 2010 & 2819 & 1089(14.93) & $290(14.46)$ & $219(16.27)$ & $50(18.12)$ & $293(21.80)$ & $878(14.49)$ & \multirow[t]{6}{*}{$<0.001$} \\
\hline 2011 & 2938 & 1144(15.69) & 309(15.39) & $221(16.42)$ & $40(14.49)$ & 245(18.23) & $979(16.16)$ & \\
\hline 2012 & 3004 & 1214(16.64) & $337(16.83)$ & 214(15.90) & $48(17.39)$ & $218(16.22)$ & $973(16.06)$ & \\
\hline 2013 & 3201 & $1268(17.39)$ & $332(16.56)$ & 263(19.54) & $36(13.04)$ & 195(14.51) & $1107(18.27)$ & \\
\hline 2014 & 3223 & $1261(17.29)$ & $355(17.71)$ & 230(17.09) & $53(19.20)$ & $215(16.00)$ & $1109(18.30)$ & \\
\hline 2015 & 3137 & 1316(18.05) & $382(19.05)$ & 199(14.78) & $49(17.75)$ & 178(13.24) & 1013(16.72) & \\
\hline \multicolumn{9}{|l|}{ Race } \\
\hline White & 13,810 & $5735(78.65)$ & $1410(70.37)$ & 997(74.04) & 193(69.93) & 1010(75.17) & 4465(73.69) & \multirow[t]{4}{*}{$<0.001$} \\
\hline Black & 3108 & $1055(14.47)$ & $422(21.02)$ & $246(18.32)$ & $65(23.55)$ & $232(17.25)$ & 1088(17.95) & \\
\hline Others & 1338 & $475(6.51)$ & $166(8.27)$ & 101(7.49) & $18(6.52)$ & $93(6.91)$ & $485(8.01)$ & \\
\hline Unknown & 66 & $27(0.37)$ & $7(0.35)$ & $2(0.15)$ & $0(0.00)$ & $9(0.67)$ & $21(0.35)$ & \\
\hline \multicolumn{9}{|l|}{ Marital status } \\
\hline Married & 7745 & $3208(43.99)$ & 748(37.30) & $615(45.62)$ & $96(34.78)$ & $602(44.76)$ & $2476(40.85)$ & \multirow[t]{3}{*}{$<0.001$} \\
\hline Unmarried & 9555 & $3719(51.01)$ & $1150(57.32)$ & 649(48.29) & 163(59.06) & $658(49.00)$ & $3216(53.08)$ & \\
\hline Unknown & 1022 & $365(5.00)$ & 107(5.38) & $82(6.08)$ & 17(6.16) & $84(6.25)$ & $367(6.07)$ & \\
\hline \multicolumn{9}{|l|}{ Grade } \\
\hline 1 & 1189 & $679(9.31)$ & $89(4.43)$ & 47(3.49) & 10(3.62) & $96(7.14)$ & $268(4.42)$ & \multirow[t]{4}{*}{$<0.001$} \\
\hline$\|$ & 5816 & $2702(37.05)$ & $489(24.35)$ & $364(27.00)$ & $48(17.39)$ & $351(26.10)$ & 1862(30.71) & \\
\hline III \& IV & 6514 & 2043(28.01) & $946(47.16)$ & 654(48.66) & 117(42.39) & 459(34.13) & 2295(37.88) & \\
\hline Unknown & 4803 & 1868(25.63) & $481(24.05)$ & $281(20.85)$ & $101(36.59)$ & 438(32.64) & 1634(27.00) & \\
\hline \multicolumn{9}{|l|}{ BC Subtype } \\
\hline $\mathrm{HR}+/ \mathrm{HER} 2-$ & 9538 & $4697(64.40)$ & 776(38.65) & 449(33.36) & $78(28.26)$ & $681(50.63)$ & 2857(47.15) & \multirow[t]{5}{*}{$<0.001$} \\
\hline HR+/HER2+ & 2497 & $858(11.76)$ & $249(12.40)$ & 287(21.32) & $36(13.04)$ & 112(8.33) & $955(15.76)$ & \\
\hline HR-/HER2+ & 1309 & 236(3.24) & 197(9.81) & 238(17.68) & $25(9.06)$ & 79(5.87) & $534(8.81)$ & \\
\hline Triple negative & 2067 & $479(6.57)$ & $426(21.22)$ & 194(14.42) & $72(26.09)$ & 183(13.61) & 713(11.77) & \\
\hline Unknown & 2911 & $1022(14.03)$ & 357(17.93) & 178(13.22) & $65(23.55)$ & $289(21.56)$ & $1000(16.50)$ & \\
\hline \multicolumn{9}{|l|}{ Stage T } \\
\hline TO & 432 & 196(2.69) & $31(1.54)$ & 26(1.93) & 13(4.71) & $63(4.68)$ & 103(1.70) & \multirow[t]{6}{*}{$<0.001$} \\
\hline $\mathrm{T} 1$ & 2156 & $971(13.33)$ & 199(9.91) & 187(13.87) & $33(11.96)$ & 179(13.31) & $587(9.68)$ & \\
\hline $\mathrm{T} 2$ & 4867 & $2179(29.88)$ & $482(24.00)$ & $454(33.75)$ & $71(25.72)$ & $300(22.38)$ & $1381(22.77)$ & \\
\hline T3 & 2584 & $1089(14.93)$ & $327(16.28)$ & 218(16.17) & 25(9.06) & 135(10.04) & $790(13.06)$ & \\
\hline $\mathrm{T} 4$ & 5222 & 1678(23.01) & $689(34.41)$ & $274(20.33)$ & $70(25.36)$ & $390(29.00)$ & $2121(35.01)$ & \\
\hline Tx & 3061 & $1179(16.17)$ & $277(13.84)$ & 188(13.95) & 64(23.19) & $277(20.59)$ & 1077(17.78) & \\
\hline \multicolumn{9}{|l|}{ Stage N } \\
\hline No & 4334 & $1987(27.25)$ & $456(22.71)$ & $338(25.15)$ & $64(23.19)$ & $369(27.43)$ & 1120(18.49) & \multirow[t]{5}{*}{$<0.001$} \\
\hline N1 & 5931 & $2508(34.40)$ & $597(29.73)$ & $519(38.58)$ & $83(30.07)$ & $377(28.10)$ & 1847(30.47) & \\
\hline N2 & 1495 & $672(9.21)$ & $161(8.07)$ & $127(9.42)$ & $22(7.97)$ & 116(8.62) & $397(6.55)$ & \\
\hline N3 & 4691 & $1416(19.42)$ & $591(29.48)$ & 253(18.77) & $67(24.28)$ & $309(22.97)$ & 2055(33.94) & \\
\hline $\mathrm{Nx}$ & 1871 & 709(9.72) & $200(10.01)$ & 109(8.09) & $40(14.49)$ & 173(12.86) & $640(10.55)$ & \\
\hline
\end{tabular}


Table 1 Patient characteristics (Continued)

\begin{tabular}{|c|c|c|c|c|c|c|c|c|}
\hline Characteristic & $n$ & $\begin{array}{l}\text { Bone } \\
\text { Metastasis (\%) }\end{array}$ & $\begin{array}{l}\text { Lung } \\
\text { Metastasis (\%) }\end{array}$ & $\begin{array}{l}\text { Liver } \\
\text { Metastasis (\%) }\end{array}$ & $\begin{array}{l}\text { Brain } \\
\text { Metastasis (\%) }\end{array}$ & $\begin{array}{l}\text { Other Metastasis } \\
\text { sites (\%) }\end{array}$ & $\begin{array}{l}\text { Multiple } \\
\text { Metastasis sites (\%) }\end{array}$ & $p$ \\
\hline \multicolumn{9}{|l|}{ Surgery } \\
\hline Yes & 4964 & 2266(31.07) & 673(33.52) & 495(36.72) & $73(26.45)$ & 464(34.50) & 993(16.38) & \multirow[t]{3}{*}{$<0.001$} \\
\hline No & 13,016 & 4874(66.83) & $1286(64.04)$ & $814(60.39)$ & $201(72.83)$ & $853(63.42)$ & 4988(82.26) & \\
\hline Unknown & 342 & $152(2.10)$ & $46(2.44)$ & $37(2.89)$ & $2(0.72)$ & $27(2.08)$ & $78(1.37)$ & \\
\hline \multicolumn{9}{|l|}{ Radiotherapy } \\
\hline Yes & 5478 & 2622(35.95) & 313(15.59) & 185(13.72) & 162(58.70) & 288(21.41) & 1908(31.46) & \multirow[t]{3}{*}{$<0.001$} \\
\hline No & 12,497 & 4509(61.84) & 1645(82.07) & 1122(83.38) & $112(40.58)$ & 1023(76.13) & 4086(67.46) & \\
\hline Unknown & 347 & $161(2.21)$ & $47(2.34)$ & $39(2.89)$ & $2(0.72)$ & $33(2.45)$ & $65(1.07)$ & \\
\hline \multicolumn{9}{|l|}{ Chemotherapy } \\
\hline Yes & 9176 & $3229(44.28)$ & 1039(51.74) & 888(65.88) & 136(49.28) & 632(46.99) & $3252(53.63)$ & \multirow[t]{2}{*}{$<0.001$} \\
\hline No/Unknown & 9146 & $4063(55.72)$ & $966(48.26)$ & $458(34.12)$ & 140(50.72) & $712(53.01)$ & $2807(46.37)$ & \\
\hline
\end{tabular}

Abbreviations: $B C$ breast cancer, $H R$ hormone receptor, $H E R 2$ human epidermal growth receptor 2, $T N$ triple negative

the first three subtypes, worst survival was seen in the brain metastasis and best survival in the bone metastasis, while in triple negative subtype, patients with multiple metastases had the worst prognoses.

\section{Cox regression analysis of survival}

In order to further figure out the effect of multiple factors on BCSS and OS, the Cox proportional hazard model was applied to the analysis. Univariable analysis of BCSS and OS proved unmarried marital status, African descent, high tumor grade, large tumor size, later $\mathrm{N}$ stage, and the Her2 positive and triple negative cancer subtypes to be distinct risk factors for poor survival (hazard ratio $[\mathrm{HR}]>1, p<0.001$ ). By contrast, married status, other race, apply of chemotherapy, radiotherapy, and surgery were found to be protective factors for better survival (hazard ratio $[\mathrm{HR}]<1, p<$ 0.001) (Table 2). As for metastatic sites, the results were consistent with those from the Kaplan-Meier analysis. Patients with bone metastasis had the best BCSS and OS, followed by patients with other, liver, lung, and multiple metastases. Specifically, patients with brain metastasis exhibited the worst BCSS, with a hazard ratio of 1.708 (95\% confidence interval $[\mathrm{CI}]=1.442-2.023, p<$ $0.001)$, and $\mathrm{OS}(\mathrm{HR}=2.492,95 \% \mathrm{CI}=2.161-2.874, p<$ 0.001 ), when compared to bone metastasis.

The variables age, race, marital status, grade, subtype, $\mathrm{T}$ stage, $\mathrm{N}$ stage, surgery, radiotherapy, chemotherapy and metastatic sites were subsequently analyzed with the multivariable Cox analysis. After adjusting for age, race/ ethnicity, marital status, tumor grade, breast cancer subtype, tumor size, nodal status, surgery, radiotherapy, and chemotherapy in the analysis, metastatic site remained an independent prognostic factor of BCSS $(p<0.001)$ and OS $(p<0.001)$. The detailed results of Cox regression analysis of BCSS and OS can be found in Table 3. Compared to patients with bone metastasis, the BCSS (HR 0.994, 95\% CI 0.881-1.122, $p=0.921$ ) and OS (HR $0.994,95 \%$ CI $0.897-1.100, p=0.902$ ) of patients with lung metastasis were not significantly different. Patients with other metastasis had similar BCSS (HR 0.955, 95\% CI $0.827-1.103, p=0.532)$ as patients with bone
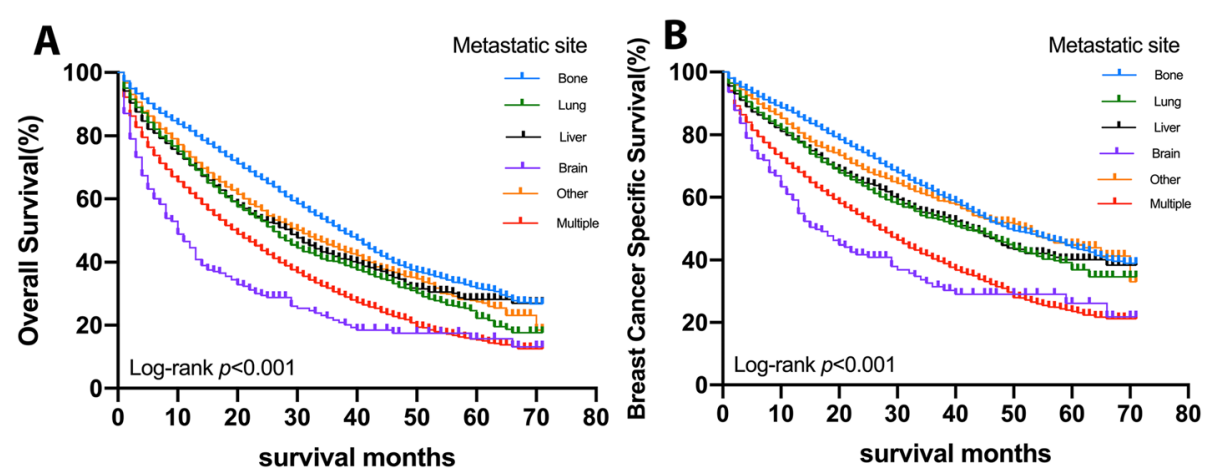

Fig. 2 Survival curves with the log-rank tests of overall survival (OS, a, $p<0.001)$ and breast cancer-specific survival $(B C S S, \mathbf{b}, p<0.001)$ based on metastatic sites for breast cancer patients 

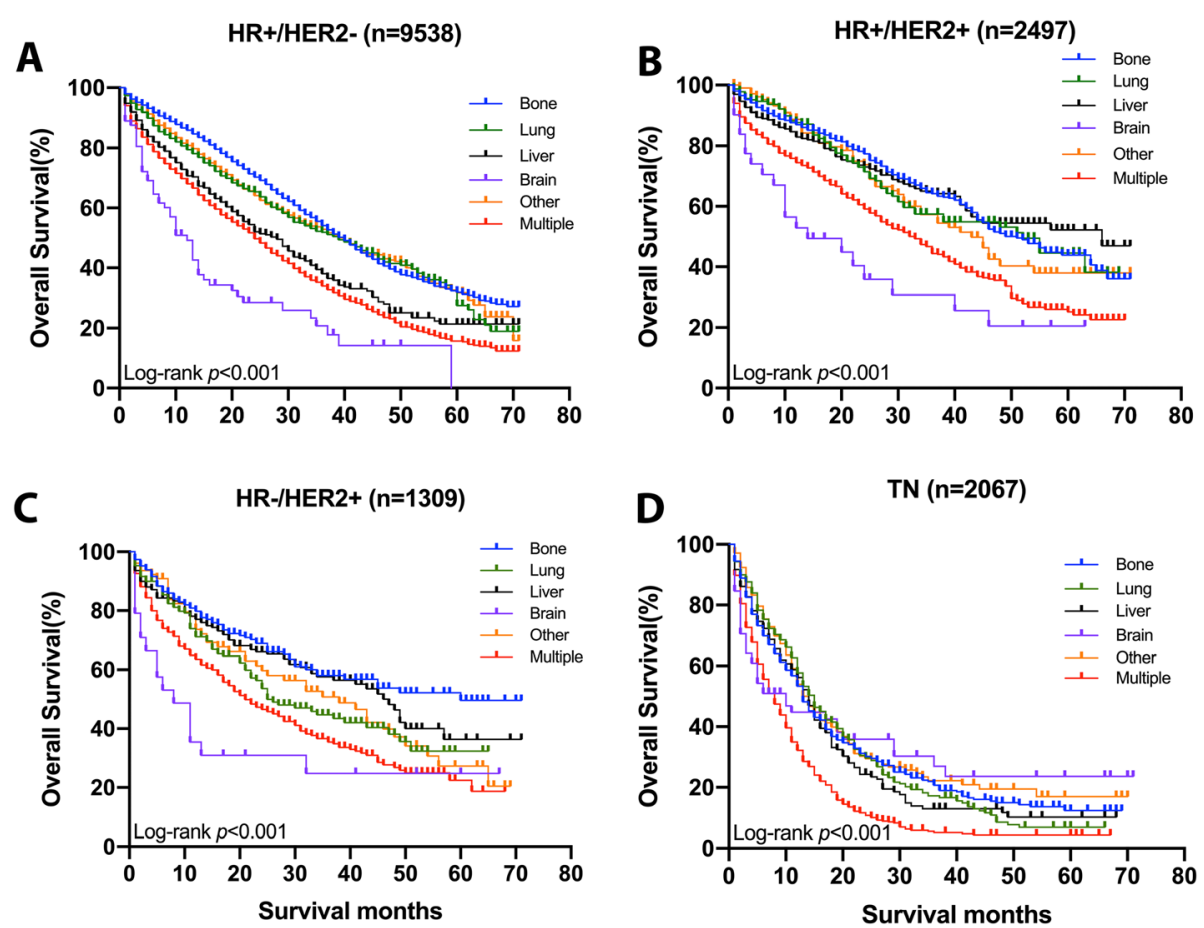

Fig. 3 Survival curves with the log-rank tests of overall survival per metastatic sites according to subtype; HR+/HER2-(a), HR+/HER2 + (b), HR -/HER2 + (c), TN (d). Abbreviations: HR: Hormone receptor, HER2: Human epidermal growth receptor 2, TN: Triple negative

metastasis, but worse OS (HR 1.127, 95\% CI 1.001$1.269, p=0.048)$. Patients with liver (HR 1.384, 95\% CI $1.208-1.586, p<0.001$; OS, HR 1.428 , 95\% CI 1.2721.602, $p<0.001$ ) and multiple metastases (BCSS, HR $1.475,95 \%$ CI 1.361-1.599, $p<0.001$; OS, HR 1.806, $95 \%$ CI 1.684-1.937, $p<0.001$ ) had worse BCSS and OS than bone metastasis. Similarly, multivariable analysis also indicated that those patients with brain metastasis had significant inferior BCSS (HR 1.975, 95\% CI 1.551-2.514, $p<0.001$ ) and OS (HR 2.307, 95\% CI 1.862-2.859, $p<$ $0.001)$ over other metastatic sites. Age $(p<0.001)$, marital status $(p<0.001)$, tumor grade $(p<0.001)$, triple negative subtype $(p<0.001)$, tumor size $(p<0.001)$, surgery $(p<0.001)$, chemotherapy $(p<0.001)$ were also statistically significant in a multivariable analysis with forward selection.

We also did a competing risk analysis to estimate the marginal probability for each cause of death. The competing risk analysis on cause of death for the entire cohort and according to metastatic sites is reported in supplementary data (Additional file 3: Figure S3). Of the cohort, the respective 5-year estimates of breast cancerspecific mortality and other cause of mortality were 56.2 and $20.7 \%$.

\section{Discussion}

In the current study, we analyzed the clinicopathological characteristics and survival of de novo metastatic breast cancer according to metastatic sites using the SEER data. Metastatic breast cancer (MBC) is widely known to have a poor prognosis compared to non-metastatic breast cancer. Bone, liver, lung, and brain are common sites of distant metastasis in breast cancer [12]. Previous studies have shown that bone is the most common distant metastatic organ in breast cancer patients [7, 13]. Similarly, our results also show that bone metastasis is the most prevalent subgroup among the study cohort, accounting for $39.8 \%$ of the total patients.

Recently published studies proposed that the metastatic patterns may differ from one another among different biological types of breast cancer. And it has also been demonstrated to have different prognostic impact on different patterns of distant metastasis. In this study, we showed that the patients with bone metastasis mainly have the $\mathrm{HR}+/ \mathrm{HER} 2$ - subtype, which is supported by several studies that patients with $\mathrm{HR}+$ are more prone to develop bone metastasis $[14,15]$. The predictive value and the relationship between visceral metastasis and breast cancer subtype are still controversial. Kennecke et al. concluded that HER2-enriched breast tumors were more likely to develop liver metastases when compared to luminal A tumors [7]. However, there are some other studies reported that liver metastasis was not associated with breast cancer subtype [16]. In this study, HR -/HER2+ tumors exhibited highest rates of liver metastasis, while $\mathrm{HR}+/ \mathrm{HER} 2-$ exhibited highest rates of lung 
Table 2 Univariate Analysis of Prognostic factors of BCSS and OS in Metastatic Breast Cancer

\begin{tabular}{|c|c|c|c|c|c|c|}
\hline \multirow[t]{2}{*}{ Characteristic } & \multicolumn{3}{|l|}{ BCSS } & \multicolumn{3}{|l|}{ OS } \\
\hline & $\overline{\mathrm{HR}}$ & $95 \% \mathrm{Cl}$ & $p$ & $\overline{H R}$ & $95 \% \mathrm{Cl}$ & $p$ \\
\hline$\overline{\text { Age }}$ & 1.017 & $1.015-1.018$ & $<0.001$ & 1.024 & $1.022-1.025$ & $<0.001$ \\
\hline \multicolumn{7}{|l|}{ Race } \\
\hline White & 1 & & & 1 & & \\
\hline Black & 1.081 & $1.019-1.147$ & 0.009 & 1.313 & $1.248-1.381$ & $<0.001$ \\
\hline Others & 0.893 & $0.813-0.98$ & 0.017 & 0.91 & $0.839-0.986$ & 0.022 \\
\hline \multicolumn{7}{|l|}{ Marital status } \\
\hline Married & 1 & & & 1 & & \\
\hline Unmarried & 1.235 & $1.177-1.297$ & $<0.001$ & 1.482 & $1.421-1.545$ & $<0.001$ \\
\hline \multicolumn{7}{|l|}{ Grade } \\
\hline I & 1 & & & 1 & & \\
\hline$\|$ & 1.154 & $1.023-1.303$ & 0.02 & 1.267 & $1.148-1.398$ & $<0.001$ \\
\hline III \& IV & 1.377 & $1.223-1.551$ & $<0.001$ & 1.824 & $1.656-2.008$ & $<0.001$ \\
\hline \multicolumn{7}{|l|}{ BC Subtype } \\
\hline $\mathrm{HR}+/ \mathrm{HER} 2-$ & 1 & & & 1 & & \\
\hline HR+/HER2+ & 1.025 & $0.949-1.108$ & 0.528 & 0.816 & $0.763-0.873$ & $<0.001$ \\
\hline HR-/HER2+ & 1.312 & $1.193-1.443$ & $<0.001$ & 1.115 & $1.027-1.212$ & $<0.001$ \\
\hline Triple negative & 1.73 & $1.616-1.852$ & $<0.001$ & 2.539 & $2.395-2.691$ & $<0.001$ \\
\hline \multicolumn{7}{|l|}{ Stage $T$} \\
\hline $\mathrm{T} 0 / \mathrm{T} 1$ & 1 & & & 1 & & \\
\hline $\mathrm{T} 2$ & 0.917 & $0.841-1.001$ & 0.052 & 1.002 & $0.935-1.074$ & 0.96 \\
\hline T3 & 0.92 & $0.836-1.012$ & 0.085 & 1.104 & $1.021-1.194$ & 0.013 \\
\hline $\mathrm{T} 4$ & 1.149 & $1.058-1.248$ & 0.001 & 1.493 & $1.397-1.595$ & $<0.001$ \\
\hline \multicolumn{7}{|l|}{ Stage N } \\
\hline NO & 1 & & & 1 & & \\
\hline N1 & 0.854 & $0.8-0.912$ & $<0.001$ & 0.892 & $0.845-0.942$ & $<0.001$ \\
\hline N2 & 0.728 & $0.662-0.8$ & $<0.001$ & 0.845 & $0.779-0.918$ & $<0.001$ \\
\hline N3 & 0.902 & $0.844-0.965$ & 0.003 & 1.096 & $1.036-1.159$ & 0.001 \\
\hline \multicolumn{7}{|l|}{ Surgery } \\
\hline No & 1 & & & 1 & & \\
\hline Yes & 0.622 & $0.588-0.659$ & $<0.001$ & 0.513 & $0.489-0.538$ & $<0.001$ \\
\hline \multicolumn{7}{|l|}{ Radiotherapy } \\
\hline No & 1 & & & 1 & & \\
\hline Yes & 0.754 & $0.717-0.793$ & $<0.001$ & 0.756 & $0.723-0.79$ & $<0.001$ \\
\hline \multicolumn{7}{|l|}{ Chemotherapy } \\
\hline No/Unknown & 1 & & & 1 & & \\
\hline Yes & 0.655 & $0.625-0.686$ & $<0.001$ & 0.626 & $0.601-0.651$ & $<0.001$ \\
\hline \multicolumn{7}{|l|}{ Metastatic site } \\
\hline Bone & 1 & & & 1 & & \\
\hline Lung & 1.319 & $1.214-1.434$ & $<0.001$ & 1.435 & $1.339-1.537$ & $<0.001$ \\
\hline Liver & 1.365 & $1.242-1.5$ & $<0.001$ & 1.415 & $1.306-1.532$ & $<0.001$ \\
\hline Brain & 1.708 & $1.442-2.023$ & $<0.001$ & 2.492 & $2.161-2.874$ & $<0.001$ \\
\hline Other & 1.127 & $1.021-1.244$ & $<0.001$ & 1.285 & $1.187-1.392$ & $<0.001$ \\
\hline Multiple & 1.484 & $1.405-1.567$ & $<0.001$ & 1.944 & $1.855-2.037$ & $<0.001$ \\
\hline
\end{tabular}

Abbreviations: $B C S S$ breast cancer-specific survival, $O S$ overall survival, $H R$ hazard ratio, $C l$ confidence interval, $B C$ breast cancer, $H R$ hormone receptor, HER2 human epidermal growth receptor $2, T N$ triple negative 
Table 3 Multivariate Analysis of Prognostic factors of BCSS and OS in Metastatic Breast Cancer

\begin{tabular}{|c|c|c|c|c|c|c|}
\hline \multirow[t]{2}{*}{ Characteristic } & \multicolumn{3}{|l|}{ BCSS } & \multicolumn{3}{|l|}{ OS } \\
\hline & $\overline{\mathrm{HR}}$ & $95 \% \mathrm{Cl}$ & $p$ & $\overline{H R}$ & $95 \% \mathrm{Cl}$ & $p$ \\
\hline$\overline{\text { Age }}$ & 1.01 & $1.008-1.013$ & $<0.001$ & 1.018 & $1.016-1.020$ & $<0.001$ \\
\hline \multicolumn{7}{|l|}{ Race } \\
\hline White & 1 & & & 1 & & \\
\hline Black & 1.074 & $0.985-1.171$ & 0.104 & 1.262 & $1.171-1.360$ & $<0.001$ \\
\hline Others & 0.932 & $0.819-1.061$ & 0.286 & 1.011 & $0.902-1.133$ & 0.85 \\
\hline \multicolumn{7}{|l|}{ Marital status } \\
\hline Married & 1 & & & 1 & & \\
\hline Unmarried & 1.1 & $1.026-1.178$ & 0.007 & 1.244 & $1.172-1.320$ & $<0.001$ \\
\hline \multicolumn{7}{|l|}{ Grade } \\
\hline I & 1 & & & 1 & & \\
\hline$\|$ & 1.199 & $1.031-1.396$ & 0.0019 & 1.353 & $1.197-1.530$ & $<0.001$ \\
\hline III \& IV & 1.372 & $1.177-1.599$ & $<0.001$ & 1.803 & $1.590-2.043$ & $<0.001$ \\
\hline \multicolumn{7}{|l|}{ BC Subtype } \\
\hline $\mathrm{HR}+/ \mathrm{HER} 2-$ & 1 & & & 1 & & \\
\hline HR+/HER2+ & 1.084 & $0.979-1.201$ & 0.12 & 0.876 & $0.800-0.959$ & 0.004 \\
\hline HR-/HER2+ & 1.363 & $1.196-1.553$ & $<0.001$ & 1.15 & $1.026-1.289$ & 0.017 \\
\hline Triple negative & 2.12 & $1.925-2.335$ & $<0.001$ & 2.823 & $2.596-3.069$ & $<0.001$ \\
\hline \multicolumn{7}{|l|}{ Stage $T$} \\
\hline $\mathrm{T} 0 / \mathrm{T} 1$ & 1 & & & 1 & & \\
\hline $\mathrm{T} 2$ & 1.019 & $0.906-1.147$ & 0.748 & 0.997 & $0.908-1.094$ & 0.948 \\
\hline T3 & 1.048 & $0.921-1.191$ & 0.478 & 1.053 & $0.948-1.170$ & 0.332 \\
\hline $\mathrm{T} 4$ & 1.189 & $1.059-1.334$ & 0.003 & 1.227 & $1.118-1.348$ & $<0.001$ \\
\hline \multicolumn{7}{|l|}{ Stage N } \\
\hline NO & 1 & & & 1 & & \\
\hline N1 & 0.923 & $0.841-1.014$ & 0.095 & 0.926 & $0.856-1.001$ & 0.054 \\
\hline N2 & 0.863 & $0.761-0.978$ & 0.021 & 1.072 & $0.962-1.194$ & 0.208 \\
\hline N3 & 0.929 & $0.844-1.023$ & 0.136 & 1.064 & $0.980-1.154$ & 0.138 \\
\hline \multicolumn{7}{|l|}{ Surgery } \\
\hline No & 1 & & & 1 & & \\
\hline Yes & 0.733 & $0.680-0.790$ & $<0.001$ & 0.595 & $0.558-0.634$ & $<0.001$ \\
\hline \multicolumn{7}{|l|}{ Radiotherapy } \\
\hline No & 1 & & & 1 & & \\
\hline Yes & 0.898 & $0.837-0.964$ & 0.003 & 0.981 & $0.922-1.042$ & 0.528 \\
\hline \multicolumn{7}{|l|}{ Chemotherapy } \\
\hline No/Unknown & 1 & & & 1 & & \\
\hline Yes & 0.685 & $0.634-0.741$ & $<0.001$ & 0.682 & $0.638-0.730$ & $<0.001$ \\
\hline \multicolumn{7}{|l|}{ Metastatic site } \\
\hline Bone & 1 & & & 1 & & \\
\hline Lung & 0.994 & $0.881-1.122$ & 0.921 & 0.994 & $0.897-1.100$ & 0.902 \\
\hline Liver & 1.384 & $1.208-1.586$ & $<0.001$ & 1.428 & $1.272-1.602$ & $<0.001$ \\
\hline Brain & 1.975 & $1.551-2.514$ & $<0.001$ & 2.307 & $1.862-2.859$ & $<0.001$ \\
\hline Other & 0.955 & $0.827-1.103$ & 0.532 & 1.127 & $1.001-1.269$ & 0.048 \\
\hline Multiple & 1.475 & $1.361-1.599$ & $<0.001$ & 1.806 & $1.684-1.937$ & $<0.001$ \\
\hline
\end{tabular}

Abbreviations: $B C S S$ breast cancer-specific survival, $O S$ overall survival, $H R$ hazard ratio, $C l$ confidence interval, $B C$ breast cancer, $H R$ hormone receptor, HER2 human epidermal growth receptor $2, T N$ triple negative 
metastasis. The brain has been previously described as a preferred site of metastasis among triple negative cancers. Martin, et al. previously reported on the incidence proportion of breast cancer patients with brain metastases from 2010 to 2013. They found that the incidence proportion of brain metastasis was highest among patients with $\mathrm{HR}-/ \mathrm{HER} 2+$ and triple-negative subtypes (11.37 and $11.45 \%$, respectively) to any distant sites at diagnosis of breast cancer [17]. Other studies based on incidence of brain involvement among patients with metastatic HER-2 positive tumor has been reported to be from 25 to $34 \%$ [18], which is significantly higher than the 3.4 and $1.9 \%$ rate for triple negative and HR -/HER2+ subtypes respectively in another study [8]. The main reason for this is that there is a very limited number of patients with brain metastasis enrolled in this study compared to other subgroups, accounting for only $1.51 \%$ of the whole cohort population, which makes it not as representative as other results. Further studies with more patients and prospective design are needed to better interpret the situation.

Some studies have shown that the survival of female breast cancer may be associated with different metastatic patterns [12, 19]. However, these studies are controversial, and there's no population-based study focus on studying the survival differences of patients with different metastatic pattern. Gerratana et al. reported that breast cancer patients with lung as the first site of distant metastasis had the best survival outcome (58.5 months) compared with those with bone ( 44.4 months), liver (36.7 months), or brain (7.35 months) as the first metastasis identified [12]. But the 5-year survival elevated significantly in patients with bone metastasis when compared with other metastasis in another study [19], which is consistent with our conclusion. In our study, the Kaplan-Meier curves showed that patients with bone metastasis had the best survival in both OS and BCSS. Lung, liver and other metastasis had very similar survival according to Kaplan-Meier analysis. Women with multiple organs affected had worse survival than those with minimal metastatic disease, a finding confirmed in this study. The presence of brain metastasis was shown in this analysis as a poor prognostic factor compared to all the other distant metastases, worse than patients with multiple metastases. We got the same result in both Kaplan-Meier survival analysis and Cox regression analysis. Taking bone metastasis as the reference, univariable and multivariable analysis showed that patients with brain metastasis had the worst prognosis. The differences of the reported outcomes may be partially due to the clinicopathological and molecular characteristics specific to different subtypes of BC. This result is in line with another study based on SEER database [17] as well as some retrospective studies [20, 21].
Of note, our study may be more comprehensively offering better guidance for the daily clinical practice, given that our analysis was based on a much larger population of $\mathrm{BC}$ patients.

Besides de novo $\mathrm{MBC}$, most $\mathrm{MBC}$ are developed from primary cancer, which is known as recurrent $\mathrm{MBC}$. The majority of $\mathrm{BC}$ patients diagnosed with early stages of disease are eligible for surgical resections and adjuvant systemic therapies, leading to longer disease-free survival. While, those develop metastatic disease during or after cycles of standard care are barely curable. A recent study conducted by Rueda et al. presented a statistical model based on distinct disease stages and competing risks of mortality to predict individual risk of distant recurrence [22]. They applied the model to a population of 3240 patients and analyzed the rates and routes of metastases and their lethality, suggesting that ER-negative patients harbored more visceral disease than the ERpositive cases. It took longer time to develop bone metastases which were more common in ER-positive than in ER-negative patients (71\% versus $43 \%$ ). They also denoted that this model was of more predictive value in predicting brain metastases incorporating the influence of the number and site of relapses on the risk of death after recurrence. We had the same conclusion in this study, which indicates that both de novo $\mathrm{MBC}$ and recurrent MBC share the same molecular characteristics and some of the biological behaviors. As for survival and prognosis, previous studies have shown that recurrent $\mathrm{MBC}$ patients tend to have a worse prognosis relative to de novo MBC patients. Yamamura et al. studied differences in survival outcomes between de novo and recurrent $\mathrm{MBC}$ patients, and found that de novo $\mathrm{MBC}$ led to a better prognosis than recurrent $\mathrm{MBC}$ with a diseasefree interval of less than 2 years or an AFI (interval from the end of adjuvant treatment to the first recurrence) less than 1 year [23]. The reason for this may be that de novo $\mathrm{MBC}$ might be more sensitive to systemic treatments for its treatment-naïve status. Recurrent $\mathrm{MBC}$, however, might be more resistant to systemic treatments after receiving adjuvant therapies. Other possible biological differences besides therapeutic factors between de novo and recurrent $\mathrm{MBC}$ patients should also be considered.

Historically, women diagnosed with metastatic breast cancer were not treated with surgery and received only systemic therapy [24]. It is believed that surgical resection of the primary tumor was palliative and performed only to relieve symptoms such as bleeding, infection, or pain. However, value of local treatment of the primary in cases of a metastatic solid tumor has been shown for metastatic renal cell carcinoma, nonfunctioning pancreatic neuroendocrine tumors and hepatocellular carcinoma [25-27]. However, there hasn't been an evidence-based consensus 
on surgery for patients with metastatic BC. A similar strategy is currently being explored in several ongoing studies for patients with MBC at initial presentation [28]. Some former retrospective data suggested no benefit from surgical resection of the primary tumor and metastases, since surgeries may increase the progression of distant metastases, in spite of a better local control of breast cancer. But an increasing number of studies suggested that surgical resection of primary tumor for highly selected stage IV patients who had favorable responses to systemic neoadjuvant chemotherapy should be considered [29,30]. This is consistent with observations from our study describing a survival benefit for both BCSS and OS of the patients accepted surgery compared to those who did not undergo surgery. The conclusion is also true when it comes to radiotherapy and chemotherapy. The multivariable Cox proportional hazards model showed both radiotherapy and chemotherapy were significantly associated with improved survival of patients in this cohort. However, the study still carries the methodological defects of a retrospective analysis, so it is impossible to derive clear recommendations. Further randomized clinical trials are needed to reach conclusions on the benefits and risks of breast local treatment including surgery and radiotherapy together with systemic treatment for women diagnosed with MBC.

In this study, age at diagnosis, race, marital status and tumor grade are prognostic factors that influenced survival besides initial sites of metastases, which is in accordance with other studies investigating prognostic factors in MBC $[8,31]$. The current analysis showed that married patients have better overall and breast cancerspecific survival compared to unmarried patients. This difference may be explained by the social psychological support given by a partner, which greatly benefits the patient. In addition to tumor characteristics as mentioned, menstrual history (age at menarche, cycle length, irregular menstruation, lifetime number of menstrual cycles, menopausal status at diagnosis and age at menopause) and reproductive factors (parity, number of fullterm pregnancy, age at first full-term pregnancy, and breastfeeding) are among the most well-established risk factors for breast cancer. A meta-analysis revealed a decrease in risk by $4-9 \%$ for each increased year of postponement in menarche, $7 \%$ for each extra birth, and $4 \%$ for every addition of 12 months of breast feeding, and an increase in risk by $3-5 \%$ each increased year of age at first birth [32]. There are also studies investigating influence of reproductive factors on tumor characteristics and breast cancer survival in women. An association had been found between early age at menarche and reduced survival. Age at menarche was also shown to be significantly associated with tumor grade and lymph node involvement $[33,34]$. Unfortunately, we are unable to do such analysis since there's no female history data in the SEER database. Further studies with focus on this point of view are needed to better delineate the picture.

With the increase of cancer incidence all over the world, a growing number of patients suffer from cancer invasion and metastasis. Metastatic breast cancer patients could be composed of heterogeneous groups and present with totally different metastatic patterns and prognostic outcomes, thus distinct treatment strategies are required. We hope to provide deeper insights into the heterogeneity of metastatic breast cancer through this population-based study. It may help to determine whether women with metastatic breast cancer after diagnosis might benefit from extended therapy to improve survival. Specifically, the brain metastasis is the most lethal subgroup of metastatic breast cancer that should be paid more attention after diagnosis, paving the way for individualized management strategies for these high-risk patient populations.

Inevitably, there are several limitations in this study that should not be overlooked. First, as a retrospective study rather than a prospective cohort study, inherent selection biases cannot be avoided and could limit the external validity of this study. Second, information about disease recurrence or subsequent sites of disease involvement is not provided in the SEER database, so that the focus of this study is de novo metastatic breast cancer, and no conclusions can be made about patients who developed metastases later in their disease course. Third, the follow-up period was relatively short with the median follow-up time of only 14 months, as the data of HER-2 status were not available until 2010. Thus, the study only focused on the short-term prognosis of metastatic breast cancer patients. These limitations may have contributed to study bias and undermined the power of analysis.

\section{Conclusion}

Despite these limitations, our results provide insight into the epidemiology, clinicopathological characteristics and survival outcomes of distant metastases in patients with newly diagnosed breast cancer in the United States. Even though patients with distant metastases are all defined as advanced cancer, the prognosis varies greatly depending on metastatic site. Patients with bone metastasis and other metastatic sites other than bone, liver, lung, or brain have better prognoses, while brain metastasis is the most aggressive group and requires additional treatment options. The metastatic sites should be taken into consideration when making therapeutic strategies for patients with advanced cancer. This knowledge is useful for the treatment of metastatic breast cancer patients in this era of individualized therapy. 


\section{Additional files}

Additional file 1: Figure S1. Survival curves with the log-rank tests of overall survival (OS, A, $p<0.001)$ and breast cancer-specific survival (BCSS, $B, p<0.001)$ based on subtype. Abbreviations: HR: Hormone receptor, HER2: Human epidermal growth receptor 2, TN: Triple negative.

Additional file 2: Figure S2. Survival curves with the log-rank tests of breast cancer-specific survival per metastatic sites according to subtype; HR+/ HER2-(A), HR+/HER2 + (B), HR-/HER2 + (C), TN(D). Abbreviations: HR: Hormone receptor, HER2: Human epidermal growth receptor 2, TN: Triple negative.

Additional file 3: Figure S3. Cumulative incidence curves of deaths to show the probability of each competing event in the entire cohort (A) and according to metastatic sites (B). The real line represents breast cancer-specific mortality and the dotted line represents competing mortality. Cumulative incidence curves of deaths to show the probability of death due to breast cancer according to metastatic sites (C).

\section{Abbreviations}

BC: Breast cancer; BCSS: Breast cancer-specific survival; Cl: Confidence interval; HER2: Human epidermal growth factor receptor 2; HR: Hazard ratio; OS: Overall survival; SEER: Surveillance, epidemiology and end results; TN: Triple negative

\section{Acknowledgements}

We thank Yupeng Yang from the Department of Surgery, Zhongshan Hospital, Fudan University, for helping us with some of the data analysis. We also thank Chelsy Chen from Johns Hopkins University, for helping us with the revision of the manuscript.

\section{Authors' contributions}

RW, JH and LN designed the study. XL1 and XL2 collected the data. RW and $Y Z$ analyzed the data. RW, $Y Z$, and $X L 1$ organized the manuscript. JH and LN reviewed the paper and revised the manuscript. All authors (RW, $Y Z, X L 1$, $\mathrm{XL2}, \mathrm{JH}$ and $\mathrm{LN}$ ) have read and approved the final manuscript. All authors contributed toward data analysis, drafting and revising the paper and agree to be accountable for all aspects of the work.

\section{Funding}

Not applicable.

\section{Availability of data and materials}

The data were abstracted from an open database, the Surveillance, Epidemiology, and End Results (SEER) database (https://seer.cancer.gov).

\section{Ethics approval and consent to participate}

This study used previously collected de-identified data, the need for informed consent had been waived due to the retrospective nature of the study, and was deemed exempt from review by the Ethics Committee of the First Affiliated Hospital of Xi'an Jiaotong University.

\section{Consent for publication}

Not applicable.

\section{Competing interests}

The authors declare that they have no competing interests.

\section{Author details}

'Department of Breast Surgery, The First Affiliated Hospital of Xi'an Jiaotong University, 277 West Yanta Road, Xi'an 710061, Shaanxi, China. ${ }^{2}$ Institute for Cell Engineering, The Johns Hopkins University School of Medicine, Baltimore, MD 21205, USA. ${ }^{3}$ Liver Cancer Institute, Zhongshan Hospital, and Key Laboratory of Carcinogenesis and Cancer Invasion (Ministry of Education), Fudan University, 180 Fenglin Road, Shanghai 200032, China.
Received: 7 July 2019 Accepted: 30 October 2019

Published online: 12 November 2019

\section{References}

1. Bray F, Ferlay J, Soerjomataram I, Siegel RL, Torre LA, Jemal A. Global cancer statistics 2018: GLOBOCAN estimates of incidence and mortality worldwide for 36 cancers in 185 countries. CA Cancer J Clin. 2018;68(6):394-424.

2. DeSantis CE, Ma J, Gaudet MM, Newman LA, Miller KD, Goding Sauer A, Jemal A, Siegel RL. Breast cancer statistics, 2019. CA Cancer J Clin:2019.

3. Noone AMHN, Krapcho M, Miller D, Brest A, Yu M, Ruhl J, Tatalovich Z, Mariotto A, Lewis DR, Chen HS, Feuer EJ, Cronin KA, editors. SEER Cancer Statistics review, 1975-2015: National Cancer Institute; 2018.

4. Howlader NNA, Krapcho M. SEER Fast Stats, 1975-2014. Stage Distrib. 2018 : 2005-14.

5. Mariotto AB, Etzioni R, Hurlbert M, Penberthy L, Mayer M. Estimation of the number of women living with metastatic breast Cancer in the United States. Cancer Epidemiol Biomarkers Prev. 2017;26(6):809-15.

6. Peart O. Metastatic breast Cancer. Radiol Technol. 2017;88(5):519M-39M

7. Kennecke $H$, Yerushalmi R, Woods R, Cheang MCU, Voduc D, Speers $\mathrm{CH}_{\text {, }}$ Nielsen TO, Gelmon K. Metastatic behavior of breast Cancer subtypes. J Clin Oncol. 2010;28(20):3271-7.

8. Lobbezoo DJ, van Kampen RJ, Voogd AC, Dercksen MW, van den Berkmortel F, Smilde TJ, van de Wouw AJ, Peters FP, van Riel JM, Peters NA, et al. Prognosis of metastatic breast cancer subtypes: the hormone receptor/HER2-positive subtype is associated with the most favorable outcome. Breast Cancer Res Treat. 2013;141(3):507-14.

9. Largillier R, Ferrero JM, Doyen J, Barriere J, Namer M, Mari V, Courdi A, Hannoun-Levi JM, Ettore F, Birtwisle-Peyrottes I, et al. Prognostic factors in 1,038 women with metastatic breast cancer. Ann Oncol. 2008;19(12):2012-9.

10. Balachandran VP, Gonen M, Smith JJ, DeMatteo RP. Nomograms in oncology: more than meets the eye. Lancet Oncol. 2015;16(4):e173-80.

11. Wingo PAJP, Hiatt RA. Building the infrastructure for nationwide cancer surveillance and control-a comparison between the National Program of Cancer registries (NPCR) and the surveillance, epidemiology, and end results (SEER) program (United States). Cancer Causes Control. 2003;14:175-93.

12. Gerratana L, Fanotto V, Bonotto M, Bolzonello S, Minisini AM, Fasola G, Puglisi F. Pattern of metastasis and outcome in patients with breast cancer. Clin Exp Metastasis. 2015;32(2):125-33.

13. Wu SG, Sun JY, Yang LC, Tang LY, Wang X, Chen XT, Liu GH, Lin HX, Lin Q, He ZY. Patterns of distant metastasis in Chinese women according to breast cancer subtypes. Oncotarget. 2016;7(30):47975-84.

14. Liede A, Jerzak KJ, Hernandez RK, Wade SW, Sun P, Narod SA. The incidence of bone metastasis after early-stage breast cancer in Canada. Breast Cancer Res Treat. 2016:156(3):587-95.

15. Kai M, Kogawa T, Liu DD, Fouad TM, Kai K, Niikura N, Hsu L, Willey JS, Theriault RL, Valero V, et al. Clinical characteristics and outcome of boneonly metastasis in inflammatory and noninflammatory breast cancers. Clin Breast Cancer. 2015:15(1):37-42.

16. Park HS, Kim S, Kim K, Yoo H, Chae BJ, Bae JS, Song BJ, Jung SS. Pattern of distant recurrence according to the molecular subtypes in Korean women with breast cancer. World J Surg Oncol. 2012;10:4.

17. Martin AM, Cagney DN, Catalano PJ, Warren LE, Bellon JR, Punglia RS, Claus EB, Lee EQ, Wen PY, Haas-Kogan DA, et al. Brain metastases in newly diagnosed breast Cancer: a population-based study. JAMA Oncol. 2017;3(8):1069-77.

18. Gaedcke J, Traub F, Milde S, Wilkens L, Stan A, Ostertag H, Christgen M, von Wasielewski R, Kreipe HH. Predominance of the basal type and HER-2/neu type in brain metastasis from breast cancer. Mod Pathol. 2007;20(8):864-70.

19. Kast K, Link T, Friedrich K, Petzold A, Niedostatek A, Schoffer O, Werner C, Klug SJ, Werner A, Gatzweiler A, et al. Impact of breast cancer subtypes and patterns of metastasis on outcome. Breast Cancer Res Treat. 2015;150(3):621-9.

20. Ording AG, Heide-Jorgensen U, Christiansen CF, Norgaard M, Acquavella J, Sorensen HT. Site of metastasis and breast cancer mortality: a Danish nationwide registry-based cohort study. Clin Exp Metastasis. 2017;34(1):93-101.

21. Gerdan L, Segedin B, Nagy V, Khoa MT, Trang NT, Schild SE, Rades D. The number of involved extracranial organs: a new predictor of survival in breast cancer patients with brain metastasis. Clin Neurol Neurosurg. 2013; 115(10):2108-10.

22. Rueda OM, Sammut SJ, Seoane JA, Chin SF, Caswell-Jin JL, Callari M, Batra R, Pereira B, Bruna A, Ali HR, et al. Dynamics of breast-cancer relapse reveal laterecurring ER-positive genomic subgroups. Nature. 2019;567(7748):399-404. 
23. Yamamura J, Kamigaki S, Fujita J, Osato H, Komoike Y. The difference in prognostic outcomes between De novo stage IV and recurrent metastatic patients with hormone receptor-positive, HER2-negative breast Cancer. In Vivo. 2018;32(2):353-8.

24. Khan SA, Stewart AK, Morrow M. Does aggressive local therapy improve survival in metastatic breast cancer? Surgery. 2002;132(4):620-6 discussion 626-627.

25. Heng DY, Wells JC, Rini Bl, Beuselinck B, Lee JL, Knox JJ, Bjarnason GA, Pal SK, Kollmannsberger CK, Yuasa T, et al. Cytoreductive nephrectomy in patients with synchronous metastases from renal cell carcinoma: results from the international metastatic renal cell carcinoma database consortium Eur Urol. 2014;66(4):704-10.

26. Keutgen XM, Nilubol N, Glanville J, Sadowski SM, Liewehr DJ, Venzon DJ, Steinberg SM, Kebebew E. Resection of primary tumor site is associated with prolonged survival in metastatic nonfunctioning pancreatic neuroendocrine tumors. Surgery. 2016;159(1):311-8.

27. Abdel-Rahman O. Role of liver-directed local tumor therapy in the management of hepatocellular carcinoma with extrahepatic metastases: a SEER database analysis. Expert Rev Gastroenterol Hepatol. 2017;11(2):183-9.

28. Ruiterkamp J, Voogd AC, Tjan-Heijnen VC, Bosscha K, van der Linden YM, Rutgers EJ, Boven E, van der Sangen MJ, Ernst MF, Dutch breast Cancer Trialists G. SUBMIT: systemic therapy with or without up front surgery of the primary tumor in breast cancer patients with distant metastases at initial presentation. BMC Surg. 2012;12:5.

29. Badwe R, Hawaldar R, Nair N, Kaushik R, Parmar V, Siddique S, Budrukkar A, Mittra I, Gupta S. Locoregional treatment versus no treatment of the primary tumour in metastatic breast cancer: an open-label randomised controlled trial. Lancet Oncol. 2015;16(13):1380-8.

30. Soran A, Ozmen V, Ozbas S, Karanlik H, Muslumanoglu M, Igci A, Canturk Z, Utkan Z, Ozaslan C, Evrensel T, et al. A randomized controlled trial evaluating resection of the primary breast tumor in women presenting with de novo stage IV breast cancer: Turkish Study (Protocol MF07-01). J Clin Oncol. 2016;34(15_suppl):1005.

31. Lobbezoo DJ, van Kampen RJ, Voogd AC, Dercksen MW, van den Berkmortel F, Smilde TJ, van de Wouw AJ, Peters FP, van Riel JM, Peters NA, et al. Prognosis of metastatic breast cancer: are there differences between patients with de novo and recurrent metastatic breast cancer? Br J Cancer. 2015;112(9):1445-51.

32. Cancer CGoHFiB. Breast cancer and breastfeeding: collaborative reanalysis of individual data from 47 epidemiological studies in 30 countries, including 50302 women with breast cancer and 96973 women without the disease. Lancet. 2002;360(9328):187-95.

33. Orgeas CC, Hall P, Rosenberg LU, Czene K. The influence of menstrual risk factors on tumor characteristics and survival in postmenopausal breast cancer. Breast Cancer Res. 2008;10(6):R107.

34. Trivers KF, Gammon MD, Abrahamson PE, Lund MJ, Flagg EW, Kaufman JS, Moorman PG, Cai J, Olshan AF, Porter PL, et al. Association between reproductive factors and breast cancer survival in younger women. Breast Cancer Res Treat. 2007;103(1):93-102.

\section{Publisher's Note}

Springer Nature remains neutral with regard to jurisdictional claims in published maps and institutional affiliations.

Ready to submit your research? Choose BMC and benefit from:

- fast, convenient online submission

- thorough peer review by experienced researchers in your field

- rapid publication on acceptance

- support for research data, including large and complex data types

- gold Open Access which fosters wider collaboration and increased citations

- maximum visibility for your research: over $100 \mathrm{M}$ website views per year

At $\mathrm{BMC}$, research is always in progress.

Learn more biomedcentral.com/submissions 